\title{
Unsteady Model for Transverse Fluid Elastic Instability of Heat Exchange Tube Bundle
}

\author{
Jun Liu, ${ }^{1,2}$ Chen Huang, ${ }^{1}$ and Naibing Jiang ${ }^{3}$ \\ ${ }^{1}$ School of Mechanical and Electrical Engineering, Southwest Petroleum University, Chengdu 610500, China \\ ${ }^{2}$ Modern Design and Simulation Lab for Oil and Gas Equipments, Southwest Petroleum University, Chengdu 610500, China \\ ${ }^{3}$ Science and Technology on Reactor System Design Technology Laboratory, Nuclear Power Institute of China, Chengdu 610041, China
}

Correspondence should be addressed to Jun Liu; 99803392@qq.com

Received 11 May 2014; Revised 11 August 2014; Accepted 12 August 2014; Published 26 August 2014

Academic Editor: Kim M. Liew

Copyright (c) 2014 Jun Liu et al. This is an open access article distributed under the Creative Commons Attribution License, which permits unrestricted use, distribution, and reproduction in any medium, provided the original work is properly cited.

\begin{abstract}
From the viewpoint of practical application, based on the unsteady analytical model for transverse fluid elastic instability of tube array proposed by Yetisir and the linear attenuation function introduced by Li Ming, a new explicit model based on nonsteady state "streamtube" hypothesis is proposed and solved using complex number method. In the model, numerical integral is avoided and inappropriate aspects in Li Ming model are modified. Using the model, the fluid elastic instability analysis of a single flexible tube is made. The stability graphs for four typical types of tube array are plotted and contrasted with experimental results. It is found that the current explicit model is effective in the analysis of transverse fluid elastic instability of tube bundle.
\end{abstract}

\section{Introduction}

Among the various fluid excitation mechanisms of the tube bundle in heat exchange, fluid elastic instability can cause the most rapid deterioration of the tube supports and has received the most attention $[1,2]$. Many efforts have been made to study this phenomenon. However the mechanism of instability is not fully understood. Connors [3] and Afshin [4] successively studied the fluid elastic instability of tube bundle induced by transverse flow with wind tunnel experiments. Based on their studies, a quasistatic model was proposed to describe fluid elastic instability and the judgment formula of critical velocity for single-phase fluid elastic instability was given. Then Blevins [5] proposed another quasistatic model for one-dimensional tube array. These models are just comprehensive analytical solutions with experimental data, not the real analytical solutions. Then Price and Paidoussis [6] and Blevins [7] put forward a quasisteady model in which the fluid influence on displacement of the tubes has been further considered. This model is the simplified form of unsteady flow model and is only suitable for high conversion velocity. Thereafter, based on the general equation of fluid mechanics, an unsteady flow model was proposed by Tanaka et al. $[8,9]$.
In the model, many parameters for the calculation of fluid elastic force need to be determined by experiment; therefore it was also known as the semiempirical theoretical model. Lever and Weaver [10] proposed a simplified elastic fluid system based on a large number of experimental observations and given analytical expressions of the fluid force used in one-dimension unsteady flow theory. Then Lai [11] used this method to study the dynamic characteristics of a heat exchange tube bundle. Potential function was also proposed by Paidoussis et al. [12] to solve the flow force and calculate the critical velocity. These models are called analytical model due to the advantages that they need less experimental data over semiempirical theory model. Furthermore, the flow force induced by the pipe movement in analytical model was derived by Yetisir and Weaver [13] using unsteady continuous and momentum equations. This method is essentially an unsteady analytical model with relatively few experimental data. The result based on the model is in good agreement with those from experiment. Consequently, many similar models were developed. However, the calculation of the flow force in this kind of model involves numerical integration. Therefore it is not very convenient in practical to use them. 


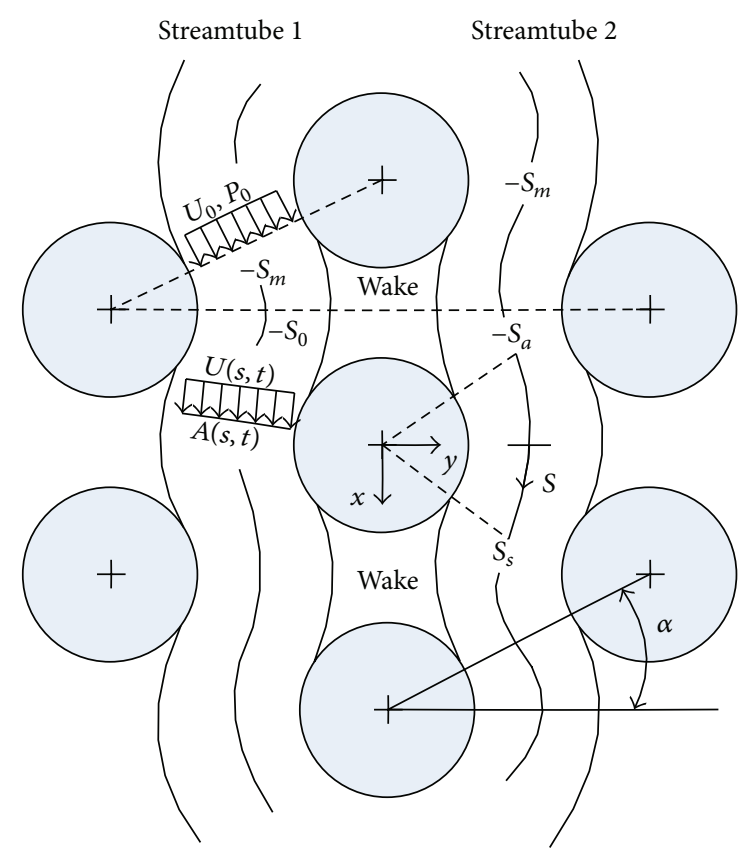

FIgURE 1: Idealized streamtube pattern [10].

In order to overcome the shortage, $\mathrm{Li}$ [14] attempted to give an explicit expression of flow force in Yetisir and Weaver's model by introducing a linear delay function. Nevertheless, there are several mistakes in his derivation such as coefficient A5. The final expression of the model is also not convenient for application.

The purpose of this paper is to present an explicit analytical model for transverse fluid elastic instability of standard array configurations which needs less experimental data and is convenient for application.

\section{Theoretical Model}

2.1. Physical Model. In Lever and Weaver's model (L-W model), "streamtube" concept is given. As shown in Figure 1, only an element of parallel triangle array is considered. On opposite sides of the centre pipe, there are two streamtubes with the sections changing with the motion of the pipe. Along the streamtubes, the curvilinear systems are set up. Meanwhile it is assumed that streamtubes began to attach to the centre flexible tube at position $s_{a}$ and separate at position $s_{s}$.

In order to derive the eventual theoretical model, the mean area of the streamtube cross section along the streamtube length is assumed constant and determined by the minimum gap area at position $-s_{l}$. Consider

$$
A_{0}=\min \left(P \cos \alpha-\frac{D}{2}, P-D\right),
$$

where $P$ is tube spacing, $D$ is tube diameter, $\alpha$ is geometric angle of tube array [13].

The instantaneous cross section area $A(s, t)$ of the streamtube changes with the vibration of the centre tube. Thus
$A(s, t)$ can be written as follows which contains average item $A_{0}$ and fluctuation item $a(s, t)$ :

$$
A(s, t)=A_{0}+a(s, t) .
$$

Similarly, velocity and pressure of the streamtube can be expressed as follows:

$$
U(s, t)=U_{0}+u(s, t), \quad P(s, t)=P_{0}+p(s, t),
$$

where $u(s, t)$ and $p(s, t)$, respectively, represent the fluctuation parts of speed and velocity. On the upper position far enough from the vibrating tube $-s_{l}$, velocity and pressure can be seen as constants $U_{0}$ and $P_{0}$.

Obviously, the vibration of the centre tube cannot immediately affect the flow at other position on the streamtube. Therefore upstream perturbation function $a(s, t)$ can be expressed as follows, based on the application of a phase function which considers the disturbance delayed effect:

$$
a(s, t)=a\left(s_{m}, t\right) f(s) e^{i \varphi(s)}
$$

where $f(s)$ is artificial attenuation function which denotes the disturbance attenuation from the flexible tube position to upstream region and $a\left(s_{m}, t\right)$ is the area perturbation at the minimum clearance position and is the function of pipe geometry.

2.2. Continuity Equation, Momentum Equation, and Pressure Equation. The application of fluid continuity equation along the streamtube can yield the following formula:

$$
\frac{\partial}{\partial t} A(s, t)+\frac{\partial}{\partial s}[A(s, t) U(s, t)]=0 .
$$

Formula (5) can also be written as follows by using the following steps: introduce formulas (2) and (3) into formula (5) with a harmonic disturbance frequency $\omega$ adopted, integrate formula (5) along the coordinate $s$ from the entrance $s=s_{i}$ to the exit $s=s_{e}$ of the streamtube, eliminate the steady state and high-order items, and normalize:

$$
\begin{aligned}
& \frac{1}{U_{r}} \frac{\omega}{\omega_{n}} \int_{s_{i}}^{s_{e}} \frac{\partial a^{*}\left(s^{*}, t^{*}\right)}{\partial t^{*}} \mathrm{~d} s^{*}+l_{0}^{*} A_{0}^{*}\left[u^{*}\left(s_{e}^{*}, t^{*}\right)-u^{*}\left(s_{i}^{*}, t^{*}\right)\right] \\
& +l_{0}^{*}\left[a^{*}\left(s_{e}^{*}, t^{*}\right)-a^{*}\left(s_{i}^{*}, t^{*}\right)\right]=0,
\end{aligned}
$$

where $U_{r}$ is reduced flow velocity and $U_{r}=U_{0} / \omega_{n} l_{0}, \omega_{n}$ is the natural frequency of the pipe in static water, $\omega$ is vibration complex frequency, $l_{0}=2 s_{1}$ ( $s_{1}$ is the distance between the vibrating tube and the position where pressure disturbance can be ignored), $t^{*}=\omega t, a^{*}(s, t)=$ $a(s, t) / D, l_{0}^{*}=l_{0} / D, A_{0}^{*}=A_{0} / D, u^{*}(s, t)=u(s, t) / U_{0}, s^{*}=$ $s / D$, and $p^{*}(s, t)=p(s, t) / P_{0}$.

Linear momentum equation of the fluid can be written as

$$
\frac{\partial}{\partial t} \int_{\forall} \mathbf{U}(s, t) \mathrm{d} \forall+\oint \rho \mathbf{U}(s, t)[\mathbf{U}(s, t) \cdot \mathbf{n}(s)] \mathrm{d} A=\frac{1}{\rho} \sum \mathbf{F},
$$




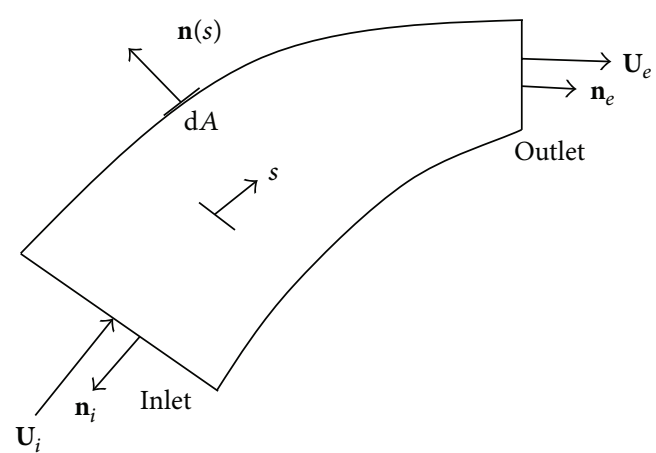

FIGURE 2: Control volume in streamtube.

where $\forall$ denotes control volume (see Figure 2) and $\forall=A \mathrm{~d} s$, $\rho$ is the fluid density, and the right item denotes the sum of external forces on the control volume with unit length.

Using the derivation process similar to the above mentioned one, the momentum equation (7) can be further written as follows by substituting formulas (2) and (3) into it:

$$
\begin{aligned}
\frac{\omega}{\omega_{n}} l_{0}^{*} U_{r} & \int_{s_{i}}^{s_{e}} \frac{\partial u^{*}\left(s^{*}, t^{*}\right)}{\partial t^{*}} \mathrm{~d} s^{*}+\frac{\omega}{\omega_{n}} \frac{l_{0}^{*}}{A_{0}^{*}} U_{r} \int_{s_{i}}^{s_{e}} \frac{\partial a^{*}\left(s^{*}, t^{*}\right)}{\partial t^{*}} \mathrm{~d} s^{*} \\
& +\left[2 l_{0}^{*} u^{*}\left(s_{i}^{*}, t^{*}\right)+\frac{l_{0}^{*}}{A_{0}^{*}} a^{*}\left(s_{i}^{*}, t^{*}\right)\right] l_{0}^{*} U_{r}^{2} \\
& +\left[2 l_{0}^{*} u^{*}\left(s_{e}^{*}, t^{*}\right)+\frac{l_{0}^{*}}{A_{0}^{*}} a^{*}\left(s_{e}^{*}, t^{*}\right)\right] l_{0}^{*} U_{r}^{2} \\
= & -p^{*}\left(s_{i}^{*}, t^{*}\right)-p^{*}\left(s_{e}^{*}, t^{*}\right) .
\end{aligned}
$$

It is worth noting that $p^{*}=p /\left(\rho d^{2} \omega_{n}^{2}\right)$ which is not mentioned in Li's model. This is probably the main reason which leads to no density $(\rho)$ existence in the final fluid force expression in Li's model.

Many studies have proved the fact that fluid elastic instability usually first occurs in the lift direction. Therefore, in the following part, only the fluid force calculation and fluid elastic analysis in the lift direction are made.

The force on the centre flexible tube can be obtained by integrating the pressure item on the pipe surface attached with fluid $\left(s_{a}^{*} \leq s \leq s_{s}^{*}\right)$. Consider

$$
F_{y}^{*}=\int_{s_{a}^{*}}^{s_{s}^{*}} p^{*}\left(s^{*}, t^{*}\right) \cos \left[\beta\left(s^{*}\right)\right] \mathrm{d} s^{*},
$$

where $\beta\left(s^{*}\right)$ is the angle of the normal vector of the tube surface and $\beta\left(s^{*}\right)=2 s^{*} / \operatorname{Pr}$. More detail about $\beta\left(s^{*}\right)$ is shown in literature [13].

2.3. Dynamic Equation. It is assumed that flexible tube vibrates in the lift and drags directions with the same harmonic oscillation

$$
m \ddot{y}+c \dot{y}+k y=F_{y} a_{1}\left(s_{m}, t\right)-F_{y} a_{2}\left(s_{m}, t\right),
$$

where $a_{1}\left(s_{m}, t\right)$ and $a_{2}\left(s_{m}, t\right)$, respectively, denote the area perturbations at the minimum clearances of streamtube 1 and streamtube 2.

The displacement mode of the centre pipe can be defined as follows:

$$
y(t)=Y e^{i \omega t},
$$

where $\omega$ is vibration complex number frequency and $Y$ is maximum vibration amplitude.

Substituting formula (11) into formula (10) and introducing the dimensionless variables, $y^{*}=Y / d, \bar{F}_{y}=F_{y} / \rho d^{3} \omega_{n}$ can yield the following equation:

$$
\begin{gathered}
\frac{m}{\rho d^{2}}\left[-\left(\frac{\omega}{\omega_{n}}\right)^{2}+\frac{\delta}{\pi}\left(\frac{\omega}{\omega_{n}}\right) i+1\right] y^{*} \\
=F_{y}^{*} a_{1}^{*}\left(s_{m}, t\right)-F_{y}^{*} a_{2}^{*}\left(s_{m}, t\right) .
\end{gathered}
$$

Formula (12) can be further transformed as follows:

$$
\begin{gathered}
\frac{m}{\rho D^{2}}=\frac{F_{y}^{*}\left(U_{r}, \omega / \omega_{n}\right)}{1-\left(\omega / \omega_{n}\right)^{2}+i(\delta / \pi)\left(\omega / \omega_{n}\right)}, \\
F_{y}^{*}=2 l_{0}^{*} U_{r} \cos \alpha F_{y}^{*}(s)=F_{R}^{*}\left(U_{r}, \frac{\omega}{\omega_{n}}\right)+i F_{l}^{*}\left(U_{r}, \frac{\omega}{\omega_{n}}\right),
\end{gathered}
$$

where $m$ is the quality of the tube with unit length (including added fluid mass), $\rho$ is fluid density, $\delta$ is damping logarithmic attenuation rate, $F_{y}^{*}$ is the fluid resultant force applied on the unit length tube in the lift direction and is the function of frequency ratio $\omega / \omega_{n}$, and reduced speed $U_{r}=U_{0} / \omega_{n} l_{0}$. It is noted that the fluid resultant force $F_{y}^{*}$ is a complex number and can be written as (13b) where $F_{R}^{*}, F_{l}^{*}$, respectively, represent real part and imaginary part.

According to the relation among the array geometry, fluid velocity at minimum interval $U_{0}$, interval fluid velocity $U_{p}=$ $[\operatorname{Pr} /(\operatorname{Pr}-1)] U_{\infty}\left(U_{\infty}\right.$ is free flow velocity, $\operatorname{Pr}$ is tube pitch ratio, and $\operatorname{Pr}=P / D)$, and fluid continuity equation, formula (14) can be obtained:

$$
U_{r}=\frac{U_{p}}{f_{n} D} \frac{1}{l_{0}^{*} A_{0}^{*}} \frac{\cos \alpha}{2 \pi}(\operatorname{Pr}-1)
$$

2.4. Area Perturbation Equation. In order to obtain the explicit expression for the calculation of fluid force, the following linear attenuation function [14] is used, according to the requirement on it. For the convenience of introduction, superscript “ $*$ " will be ignored in the following derivation process:

$$
\begin{gathered}
f(s)=\frac{s-s_{1}}{s_{a}-s_{1}}, \quad s_{1} \leq s \leq s_{a}, \\
f(s)=1, \quad s_{a} \leq s \leq s_{s} .
\end{gathered}
$$


Noting the fact that perturbation attenuation only takes place on "streamtube" path, two area perturbation equations for parallel triangular tube array are presented as follows:

$$
\begin{array}{r}
a_{1}\left(s, U_{r}\right)=f(s) e^{i \varphi\left(s, U_{r}\right)} \cos \alpha, \\
\varphi\left(s, U_{r}\right)=\varphi\left(U_{r}\right) \frac{s-s_{a}}{s_{1}-s_{a}}, \\
s_{1} \leq s \leq s_{a}, \\
a_{2}=\cos \alpha, \quad f(s)=1.0, \quad \varphi\left(s, U_{r}\right)=0, \\
s_{a} \leq s \leq s_{s} .
\end{array}
$$

For other types of tube array, area perturbation equations are similar as the above equations except for the item $\cos \alpha$.

2.5. Solving Method. For the convenience of derivation, let $\bar{s}=\left(s-s_{a}\right) /\left(s_{1}-s_{a}\right)$, so that attenuation function and (16) can be rewritten, respectively, as follows:

$$
\begin{gathered}
f(s)=1-\bar{s}, \\
a_{1}\left(s, U_{r}\right)=a\left(-s_{m}, t\right) f(s) e^{i \varphi(s)} \\
=\frac{Y}{D} e^{i \omega t} \cos \alpha(1-\bar{s}) e^{i \bar{s} \varphi\left(U_{r}\right),} \\
s_{1} \leq s \leq s_{a}, \\
a_{2}=\frac{Y}{D} e^{i \omega t} \cos \alpha, \quad f(s)=1.0, \quad \varphi\left(s, U_{r}\right)=0, \\
s_{a} \leq s \leq s_{s} .
\end{gathered}
$$

To further facilitate the derivation, $\varphi\left(U_{r}\right)$ is briefly written as $\varphi$ in the following work.

Substituting (17) into (6) yields the following expression:

$$
u_{1}\left(s, U_{r}\right)=\left(A_{1} e^{i \varphi \bar{s}}+A_{2} \bar{s} e^{i \varphi \bar{s}}+A_{3}\right) \frac{Y}{D} e^{i \omega t} \cos \alpha,
$$

where

$$
\begin{aligned}
& A_{1}=-\frac{1}{A_{0}}-\frac{\omega_{r}}{l_{0} A_{0} U_{r}}\left(s_{l}-s_{a}\right)\left(\frac{1}{\varphi}-\frac{i}{\varphi^{2}}\right), \\
& A_{2}=\frac{1}{A_{0}}+\frac{\omega_{r}}{l_{0} A_{0} U_{r}}\left(s_{l}-s_{a}\right) \frac{1}{\varphi}, \\
& A_{3}=-\frac{\omega_{r} i}{l_{0} A_{0} U_{r}}\left(s_{1}-s_{a}\right) \frac{1}{\varphi^{2}} e^{i \varphi} .
\end{aligned}
$$

In the same way we have

$$
\begin{array}{r}
u_{2}\left(s, U_{r}\right)=\left(A_{4} \bar{s}+A_{5}\right) \frac{Y}{D} e^{i \omega t} \cos \alpha, \\
0 \leq \bar{s} \leq \frac{s_{s}-s_{a}}{s_{1}-s_{a}},
\end{array}
$$

where

$$
\begin{aligned}
& A_{4}=-\frac{\omega_{r} i}{l_{0} A_{0} U_{r}}\left(s_{l}-s_{a}\right), \\
& A_{5}=-\frac{\omega_{r}}{l_{0} A_{0} U_{r}}\left(s_{l}-s_{a}\right)\left(\frac{1}{\varphi}-\frac{i}{\varphi^{2}}+\frac{i}{\varphi^{2}} e^{i \varphi}\right)-\frac{1}{A_{0}} .
\end{aligned}
$$

Substituting (18) and (19b) into the integral expression for unsteady pressure (8) yields the following formula:

$$
p_{2}\left(s, U_{r}\right)=\left(C_{1} \bar{s}^{2}+C_{2} \bar{s}+C_{3}\right) \frac{Y}{D} e^{i \omega t} \cos \alpha,
$$

where

$$
\begin{aligned}
& C_{1}=-\omega_{r} l_{0} U_{r} i B_{2}, \\
& C_{2}=-\omega_{r} l_{0} U_{r} i B_{3}-2 l_{0}^{2} U_{r}^{2} A_{4}-\frac{\omega_{r} l_{0} U_{r} i}{A_{0}}\left(s_{1}-s_{a}\right), \\
& C_{3}=-\omega_{r} l_{0} U_{r} i B_{1}-2 l_{0}^{2} U_{r}^{2} A_{5}-\frac{l_{0}^{2} U_{r}^{2}}{A_{0}}-\frac{\omega_{r} l_{0} U_{r}}{A_{0}} B_{4},
\end{aligned}
$$

where

$$
\begin{aligned}
B_{1}= & \left(s_{1}-s_{a}\right) \\
& \times\left(\frac{-i A_{1}}{\varphi}+\frac{i A_{1}}{\varphi} e^{i \varphi}+\frac{i A_{2}}{\varphi} e^{i \varphi}+\frac{A_{2}}{\varphi^{2}}-\frac{A_{2}}{\varphi^{2}} e^{i \varphi}-A_{3}\right), \\
B_{2}= & \frac{\left(s_{1}-s_{a}\right) A_{4}}{2}, \\
B_{3}= & \left(s_{1}-s_{a}\right) A_{5}, \\
B_{4}= & \left(s_{1}-s_{a}\right)\left(-\frac{i}{\varphi}-\frac{1}{\varphi^{2}}+\frac{1}{\varphi^{2}} e^{i \varphi}\right) .
\end{aligned}
$$

The explicit expression for the fluid force applied on the centre moving tube is obtained by substituting formula (21) into formula (9). Consider

$$
\begin{aligned}
F_{y} & =\frac{Y}{D} e^{i \omega t} \cos \alpha \int_{s_{a}}^{s_{s}} p_{2}\left(s, U_{r}\right) \cos \left(\frac{2 s}{\mathrm{Pr}}\right) \mathrm{d} s \\
& =\frac{Y}{D} e^{i \omega t} \cos \alpha \int_{s_{a}}^{s_{s}}\left(C_{1} \bar{s}^{2}+C_{2} \bar{s}+C_{3}\right) \cos \left(\frac{2 s}{\mathrm{Pr}}\right) \mathrm{d} s \\
& =\frac{Y}{D} e^{i \omega t} \cos \alpha \sum_{i=1}^{3} C_{i} D_{i},
\end{aligned}
$$


TABLE 1: Numerical values of geometric parameters for test arrays [1].

\begin{tabular}{lcccccccc}
\hline Layout pattern & $P / d$ & $\delta$ & $\rho\left(\mathrm{kg} / \mathrm{m}^{3}\right)$ & $A_{0} / d$ & $s_{0} / d$ & $s_{1} / d$ & $l_{0} / d$ & $x_{1} / d$ \\
\hline \multirow{3}{*}{ Square $\left(90^{\circ}\right)$} & 1.25 & 0.012 & 1.112 & 0.25 & 1.25 & 1.25 & 5.00 & 0.05 \\
& 1.32 & 0.015 & 1.125 & 0.32 & 1.32 & 1.32 & 5.28 & 0.05 \\
& 1.41 & 0.010 & 1.110 & 0.41 & 1.41 & 1.41 & 5.64 & 0.05 \\
\hline \multirow{2}{*}{ Rotated triangular $\left(60^{\circ}\right)$} & 1.25 & 0.024 & 1.124 & 0.25 & 0.654 & 0.982 & 2.616 & 0.104 \\
& 1.32 & 0.021 & 1.110 & 0.32 & 0.691 & 1.037 & 2.764 & 0.0985 \\
& 1.41 & 0.030 & 1.110 & 0.41 & 0.738 & 1.107 & 2.952 & 0.092 \\
\hline \multirow{2}{*}{ Rotated square $\left(45^{\circ}\right)$} & 1.25 & 0.019 & 1.110 & 0.25 & 0.981 & 1.472 & 3.925 & 0.155 \\
& 1.41 & 0.015 & 1.110 & 0.41 & 1.107 & 1.660 & 4.427 & 0.137 \\
\hline \multirow{2}{*}{ Triangular $\left(30^{\circ}\right)$} & 1.25 & 0.014 & 1.110 & 0.125 & 1.249 & 1.874 & 4.996 & 0.203 \\
& 1.41 & 0.018 & 1.110 & 0.205 & 1.371 & 2.057 & 5.484 & 0.181 \\
\hline
\end{tabular}

where

$$
\begin{aligned}
D_{1}= & \left.\left(\frac{1}{s_{1}-s_{a}}\right)^{2}\left(\frac{2 s}{b^{2}} \cos b s-\frac{2}{b^{3}} \sin b s+\frac{s^{2}}{b} \sin b s\right)\right|_{s_{a}} ^{s_{s}} \\
& -\left.\frac{2 s_{a}}{\left(s_{1}-s_{a}\right)^{2}}\left(\frac{1}{b^{2}} \cos b s+\frac{s}{b} \sin b s\right)\right|_{s_{a}} ^{s_{s}} \\
& +\left.\left(\frac{s_{a}}{s_{1}-s_{a}}\right)^{2} \frac{1}{b} \sin b s\right|_{s_{a}} ^{s_{s}}, \\
D_{2}= & \left.\frac{1}{s_{1}-s_{a}}\left(\frac{1}{b^{2}} \cos b s+\frac{s}{b} \sin b s\right)\right|_{s_{a}} ^{s_{s}} \\
& -\left.\frac{s_{a}}{s_{1}-s_{a}} \frac{1}{b} \sin b s\right|_{s_{a}} ^{s_{s}}, \\
D_{3}= & \int_{s_{a}}^{s_{s}} \cos \left(\frac{2 s}{\operatorname{Pr}}\right) \mathrm{d} s \\
= & \int_{s_{a}}^{s_{s}} \cos (b s) \mathrm{d} s=\left.\frac{1}{b} \sin b s\right|_{s_{a}} ^{s_{s}},
\end{aligned}
$$

where

$$
a=\frac{\varphi}{s_{1}-s_{a}}, \quad b=\frac{2}{\operatorname{Pr}} .
$$

In formula (24), $F_{y}$ is fluid force with complex number form and can be written as $F_{y}=F_{R}+F_{I} i$. By substituting the fluid force into formula (13a), the relation between the reduced fluid velocity $U_{p} / f_{n} D$ and quality-damping ratio $m \delta / \rho D^{2}$ can be obtained.

\section{Example}

Using the above explicit unsteady model, fluid elastic instability analyses on four standard tube arrays are carried out and the result is compared with those from experiment in order to verify the validity of the model. Geometric parameters of the four types of tube arrays are listed in Table 1. The diameter and first order natural frequency of the tubes in experiment are, respectively, $0.022 \mathrm{~m}$ and $90 \mathrm{~Hz}$.

The results from the explicit analytic method and experiment are presented in Figure 3 where vertical coordinate denotes reduced fluid velocity and horizontal coordinate denotes quality-damping parameter. It is shown in the figure that for the four typical tube arrays, the results from the explicit analytic method are consistent with those from Nie's experiment [1]. For $m \delta / \rho D^{2}>5$, the results based on current method tend to be more conservative than the result of experiment, whereas, for $m \delta / \rho D^{2}<5$, the result of experiment is more conservative. But the difference between the two types of results is not great. The influence of the pitch ratio $(P / d)$ on the result is not obvious. This is consistent with the observed phenomenon by Lever and Weaver [10].

It is shown in the above analysis that the current explicit analytic method inherits the advantage of Y-W "streamtube" model which possesses good calculation precision and needs little experiment data. Moreover, comparing with Y-W model, the unsteady explicit model presented in this paper has another advantage that no numerical integral is needed in the calculation of fluid force due to its explicit expression. Therefore, the current method is more convenient for practical application.

\section{Conclusion}

This paper introduces an unsteady model in detail for fluid elastic instability analysis of standard array configuration in transverse flow. Complex number solving method is used to deduce the unsteady explicit analytical model with new area perturbation equation. In the model, numerical integration similar to Y-W model is avoided and inappropriate parameters presented in Li's model are corrected. Finally the explicit expression of the model which is convenient for application is presented.

Fluid elastic instability analysis on four standard array configurations is made and the stability maps based on current model are given. A comparison of the result between the current model and those from Nie's experiment data is 


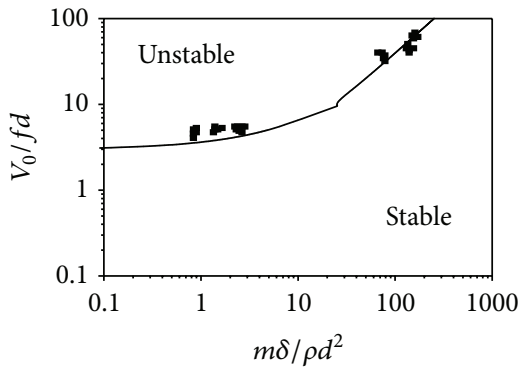

(a) Square arrays with $P / d=1.25$

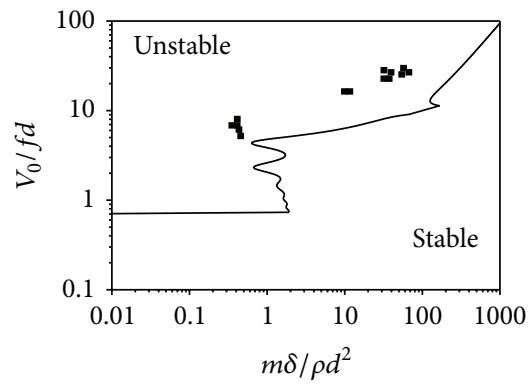

(d) Triangular arrays with $P / d=1.25$

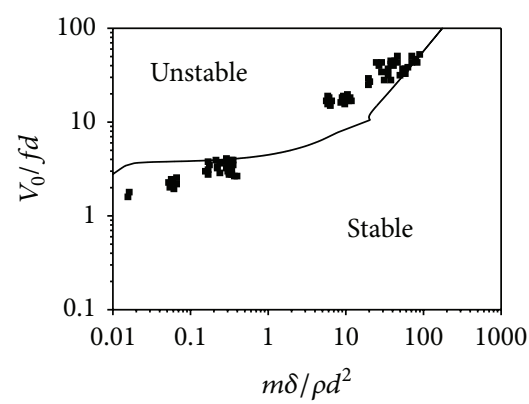

(g) Square arrays with $P / d=1.41$

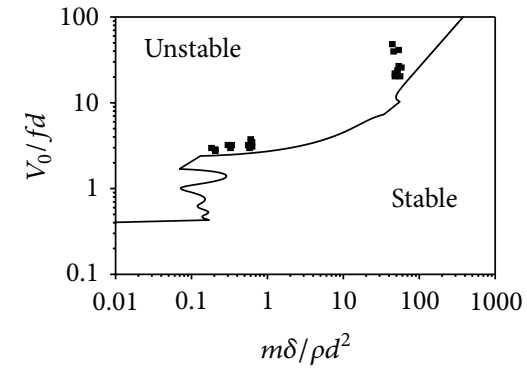

(b) Rotated triangular arrays with $P / d=1.25$

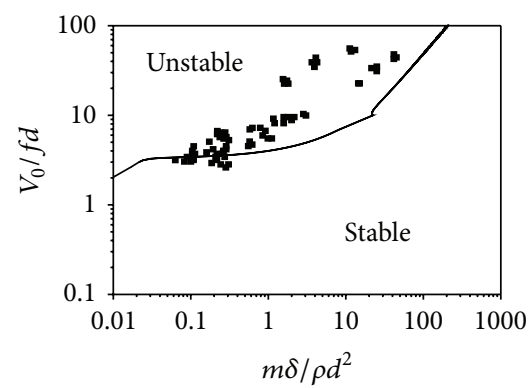

(e) Square arrays with $P / d=1.32$

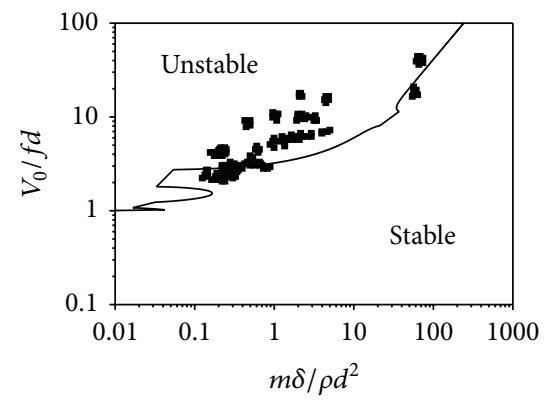

(h) Rotated triangular arrays with $P / d=1.41$

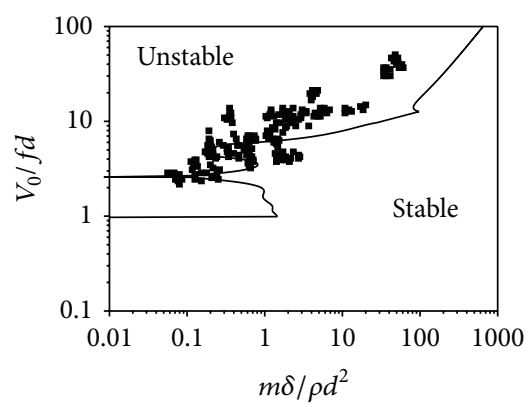

(j) Triangular arrays with $P / d=1.41$

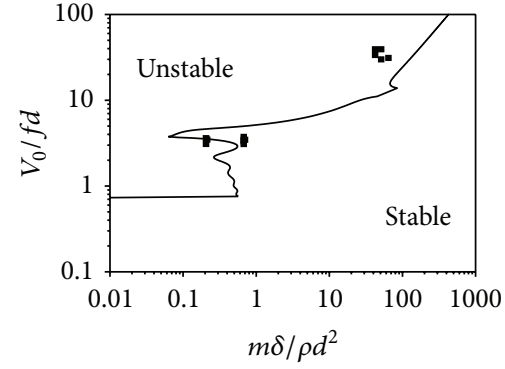

(c) Rotated square arrays with $P / d=1.25$

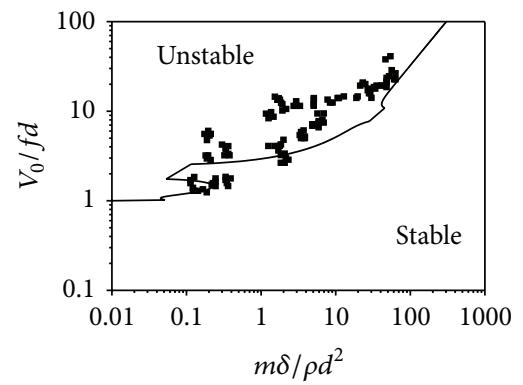

(f) Rotated triangular arrays with $P / d=1.32$

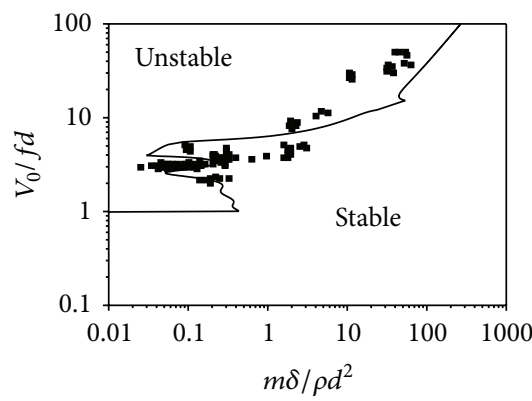

(i) Rotated square arrays with $P / d=1.41$

FIGURE 3: Stability maps for typical tube arrays (solid black points represent Nie's experiment result and black solid lines represent the result in this paper).

made to indicate the validity of current explicit fluid elastic analytical model.

\section{Conflict of Interests}

The authors declare that there is no conflict of interests regarding the publication of this paper.

\section{Acknowledgments}

This research has been supported by the National Natural Science Foundation of China (nos. 51105319 and 51274171), National Significant Science and Technology Special Subproject of China (2011ZX05026-001-07), and National Key Laboratory of Nuclear Reactor System Design Technology Foundation of China. 


\section{References}

[1] Q. D. Nie, B. Y. Guo, and X. R. Ding, "Fluidelastic instability of tube bundle in heat exchanger," Chinese Journal of Theoretical and Applied Mechanics, vol. 28, no. 2, pp. 151-158, 1996.

[2] D. L. Wen, "Study on the vibration design and improvement for tube bundle in tube-shell heat exchanger," Refrigeration \& AirCondition, vol. 10, no. 2, pp. 35-37, 2010.

[3] H. Connors, Fluidelastic Vibration of Tube Arrays Excited by Cross Flow, Flow-Induced Vibration in Heat Exchangers, 1970, edited by D. D. Reiff, ASME, New York, NY, USA, 1970.

[4] K. Afshin, Effect of Preferential Flexibility Angle on Fluidelastic Instability of a Rotated Triangular Tube Bundle, University De Montreal, 2007.

[5] R. D. Blevins, Vibration Induced by Fluid, China Machine Press, 1982.

[6] S. J. Price and M. P. Paidoussis, "Fluidealstic instability of an infinite double row of acoustics circular cylinders subjected to a uniform cross flow," Journal of Vibration, Acoustics, Stress, and Reliability in Design, vol. 105, no. 1, pp. 59-66, 1983.

[7] R. D. Blevins, "Fluid damping and the whirling instability of tube arrays," in Flow-Induced Vibrations, pp. 35-39, ASME, New York, NY, USA, 1979.

[8] H. Tanaka, S. Takahara, and K. Ohta, "Flow-induced vibration of tube arrays with various pitch-to diameter ratio," Journal of Pressure Vessel Technology, vol. 104, no. 3, pp. 168-174, 1982.

[9] N. W. Mureithi, T. P. Sawadogo, and A. A. Grie, "Estimation of the fluid-elastic instability boundary for steam-generator tubes subjected to two-phase flows," in Proceedings of the 18th International Conference on Nuclear Engineering (ICONE '10), Paper no. ICONE18-30241, pp. 679-690, Xian, China, May 2010.

[10] J. H. Lever and D. S Weaver, "On the stability of heat exchanger tube bundles, part I: modified theoretical model.Part II: numerical results and comparison with experiments," Sound and Vibration, vol. 107, no. 1, pp. 375-410, 1986.

[11] Y. X. Lai, Analysis on dynamic characteristic and flow induced vibration of heat exchanger tube bundles [Doctor thesis], Nanjing University of Technology, 2006.

[12] M. P. Paidoussis, D. Mavriplis, and S. J. Price, "A potential flow theory for the dynamics of cylinder arrays in cross flow," Journal of Fluid Mechanics, vol. 146, pp. 227-252, 1984.

[13] M. Yetisir and D. S. Weaver, "An unsteady theory for fluidelastic instability in an array of flexible tubes in cross-flow, Part I: theory," Journal of Fluids and Structures, vol. 7, no. 7, pp. 751766, 1993.

[14] M. Li, An experimental and theoretical study of fluidelastic instability in cross flow multi-span heat exchanger tube arrays [Doctor thesis], McMaster University, 1997. 


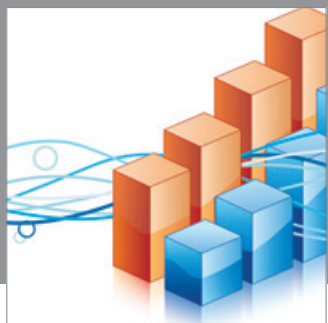

Advances in

Operations Research

mansans

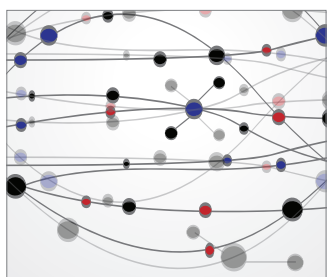

The Scientific World Journal
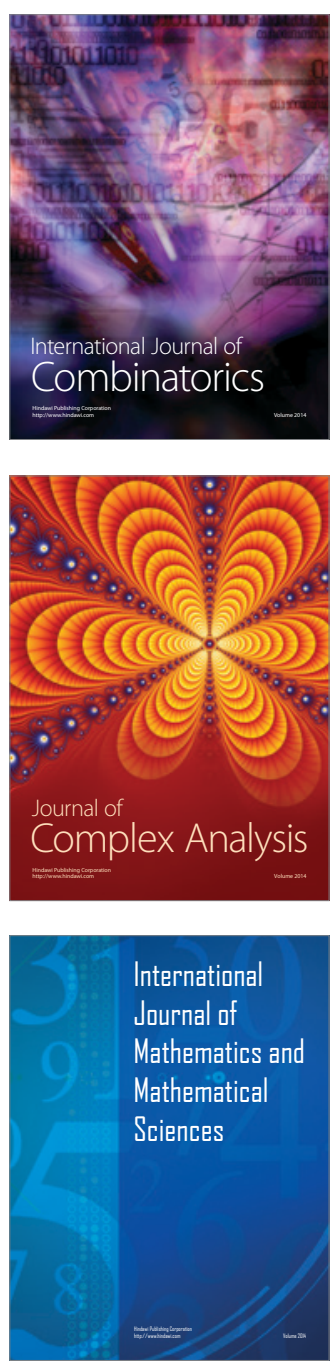
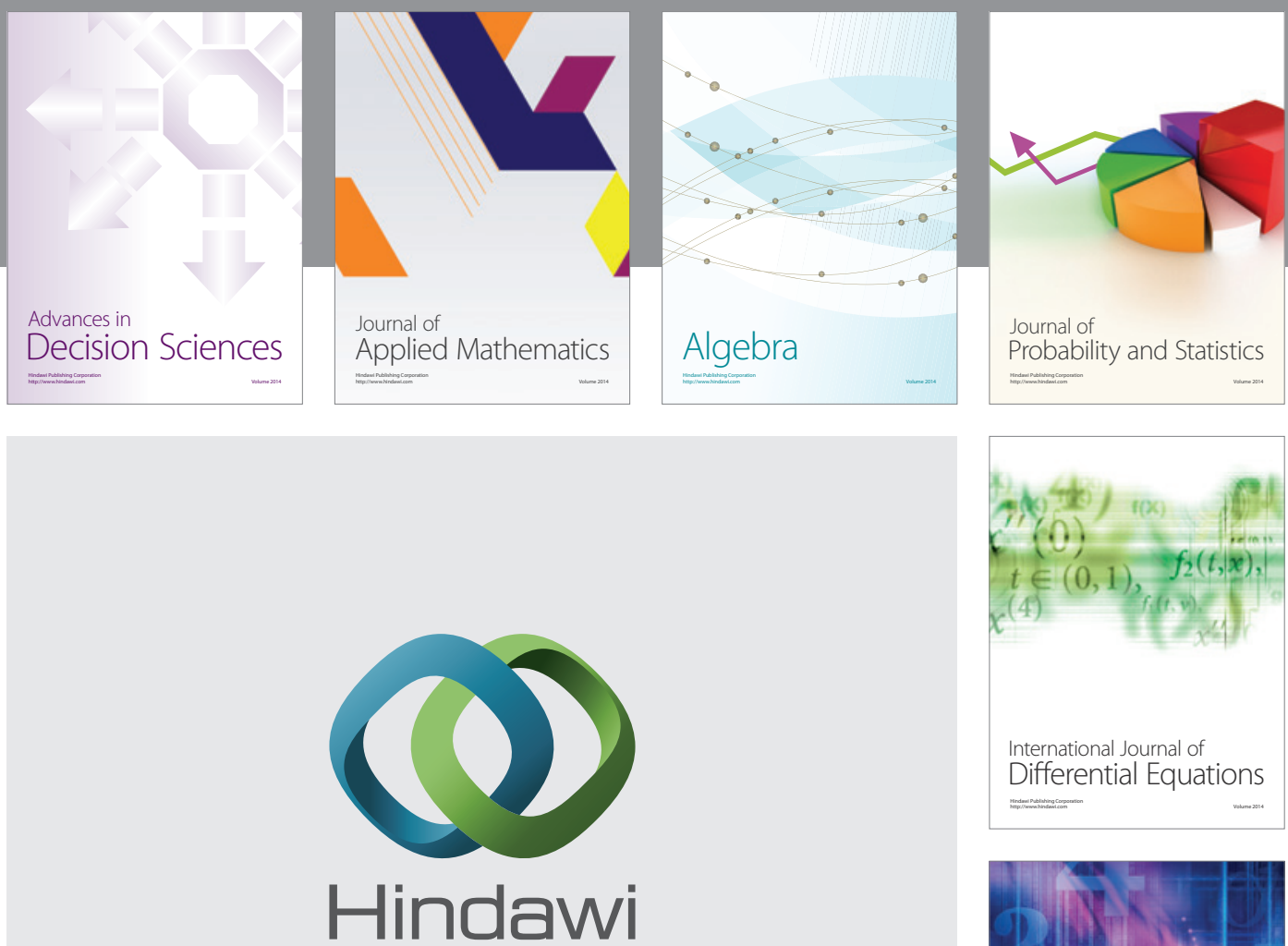

Submit your manuscripts at http://www.hindawi.com
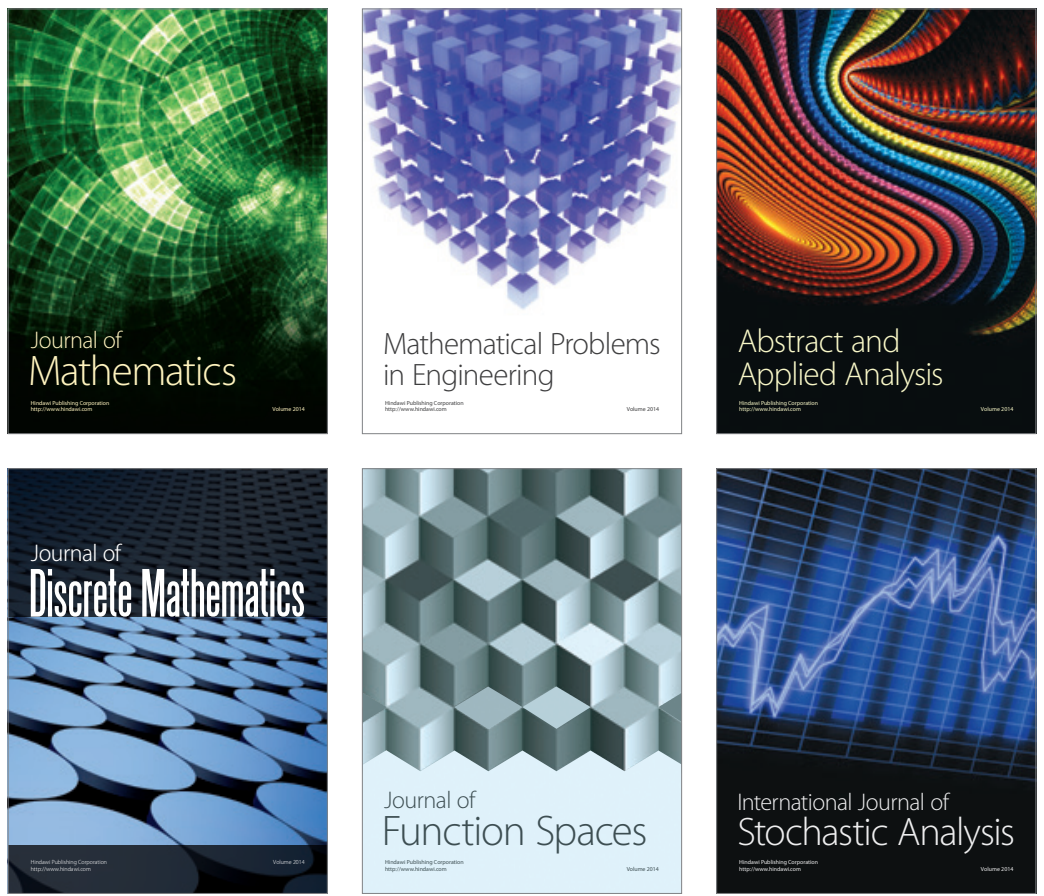

Journal of

Function Spaces

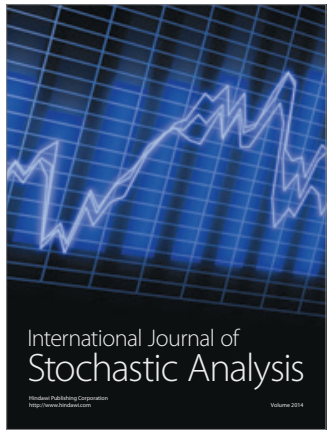

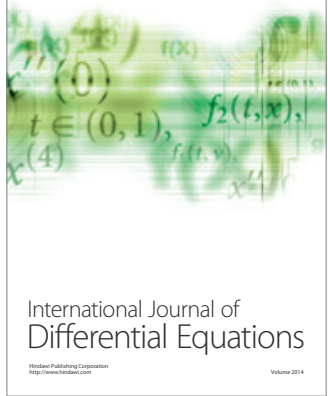
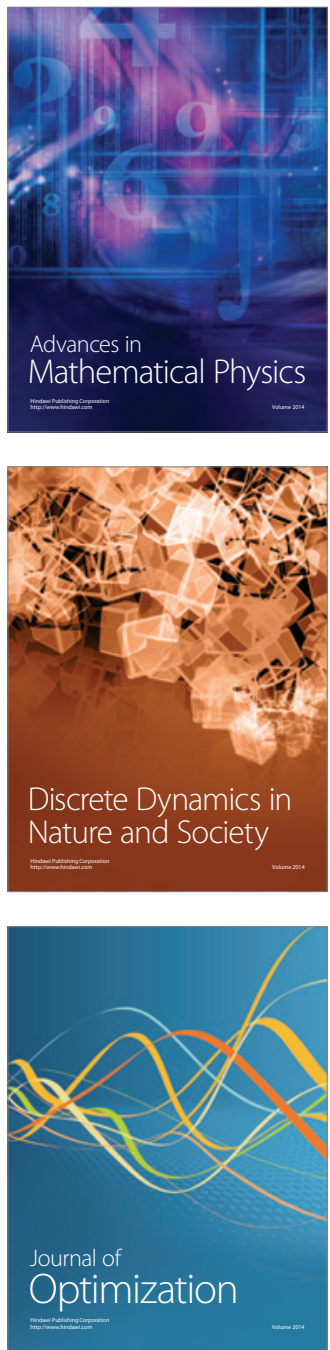